\title{
Simulation of hemispherical transducers for transcranial HIFU treatments using the hybrid angular spectrum approach
}

\author{
Scott Almquist*, Dennis Parker, Douglas Christensen \\ From Current and Future Applications of Focused Ultrasound 2014. 4th International Symposium \\ Washington, D.C, USA. 12-16 October 2014
}

\section{Background/introduction}

Transcranial focused ultrasound is emerging as a promising treatment modality for a variety of disorders including essential tremor and glioblastoma. However, traditional full-wave methods of simulating ultrasound pressure fields for these treatments, such as FDTD, are computationally intensive. The situation is exacerbated by the large area insonified by the transcranial transducers, which are typically hemispherical to spread the intensity over a large area of the skull and prevent burns. The Hybrid Angular Spectrum (HAS) approach [1] has been used to rapidly model ultrasonic beam propagation.

Here we present a method for adapting the HAS technique to hemispherical transducers.

\section{Methods}

The HAS approach assumes that the pressure pattern incident on the front face of the 3D rectangular model is defined on a plane nominally perpendicular to the propagation axis of the transducer. This is problematic for a hemispherical transducer that wraps around the model. To circumvent this limitation, we divide the transducer into seven sections of spatially clustered elements. For each section, the elements, along with the acoustic model, are rotated into a coordinate system with the direction of propagation away from the center of the section. The HAS method is applied using the specific elements of the section, then the resulting pressure pattern is rotated and interpolated back to the original coordinate frame. The fully insonified field is the superposition of the resulting pressure patterns from each section. The resulting field can be used in temperature simulations or to verify the usefulness of phase corrections. Simulations of a 1024element $650-\mathrm{kHz}$ InSightec ExAblate transducer were carried

University of Utah, Salt Lake City, Utah, United States out using this method on a selected set of retrospective clinical data. The acoustic speed of sound, density and attenuation values for the skull were derived from a CT scan with $0.43 \times 0.43 \times 1.0-\mathrm{mm}$ resolution using a previously published method of conversion [2]. The simulated skull/brain model contained $512 \times 512 \times 195$ voxels.

\section{Results and conclusions}

The average calculation time for each of the sections, including rotation of the model, propagation, and backwards rotation and interpolation of the pressure field,

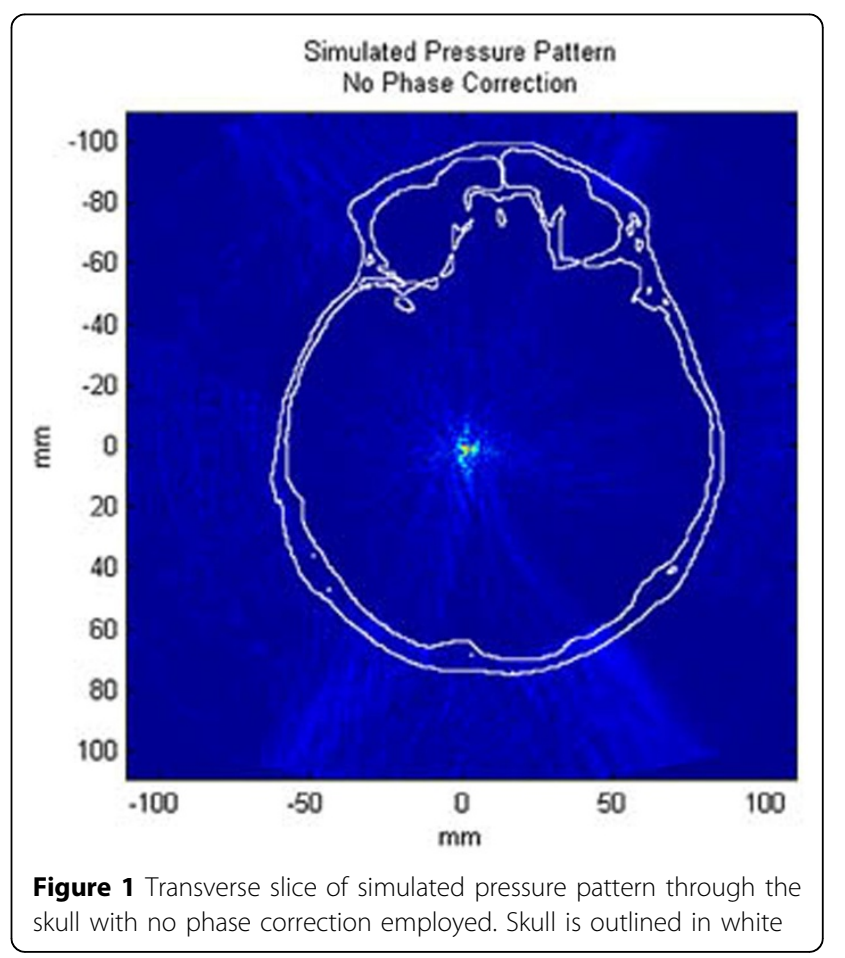




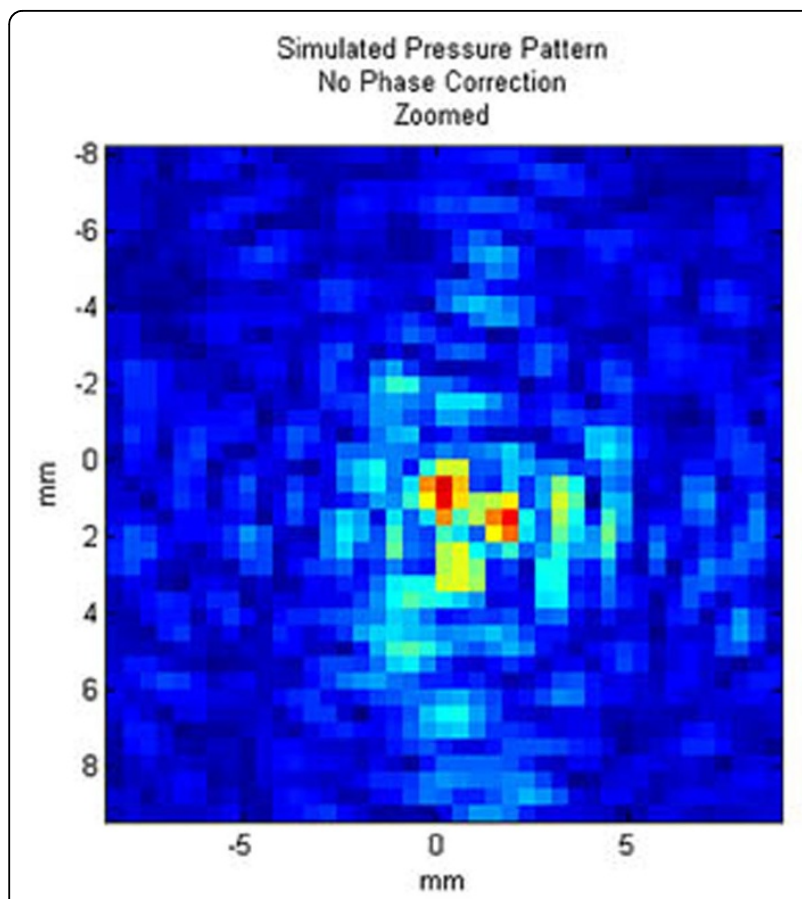

Figure 2 Zoomed-in view of focal spot for pressure pattern shown in $1 \mathrm{a}$.

was under 14.5 minutes. Figure 1 shows a transverse slice at the location of peak pressure for one clinical case; Figure 2 shows a zoomed-in view of the focal point. Figures 3-4 present the same pressure field using the experimentally obtained phase corrections.

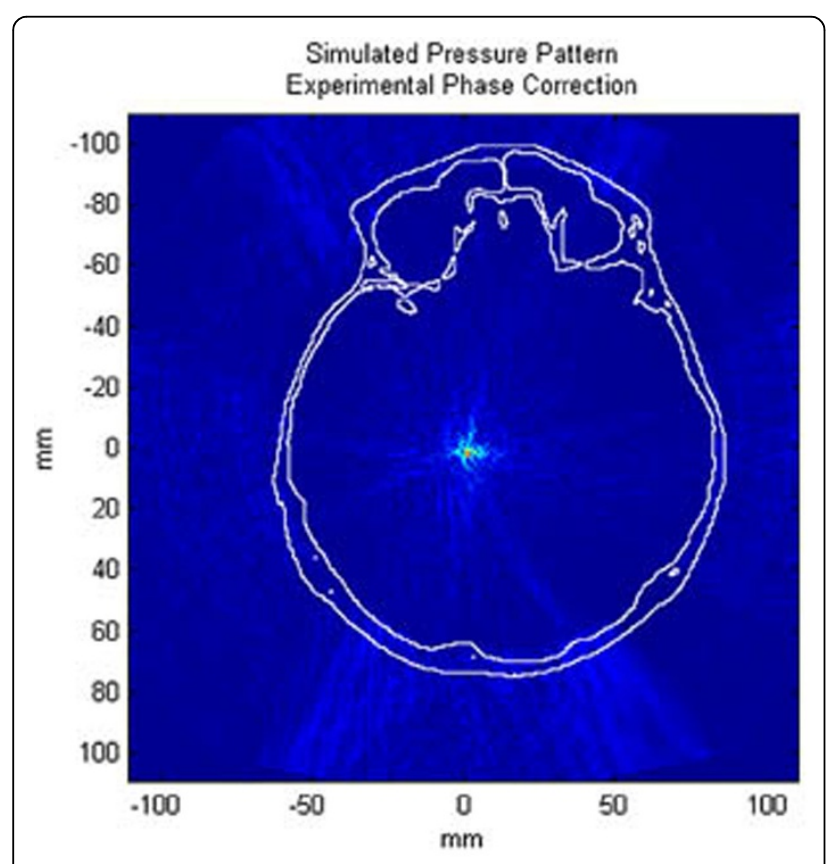

Figure 3 Transverse slice of simulated pressure pattern through the skull with experimentally employed phase correction. Skull is outlined in white

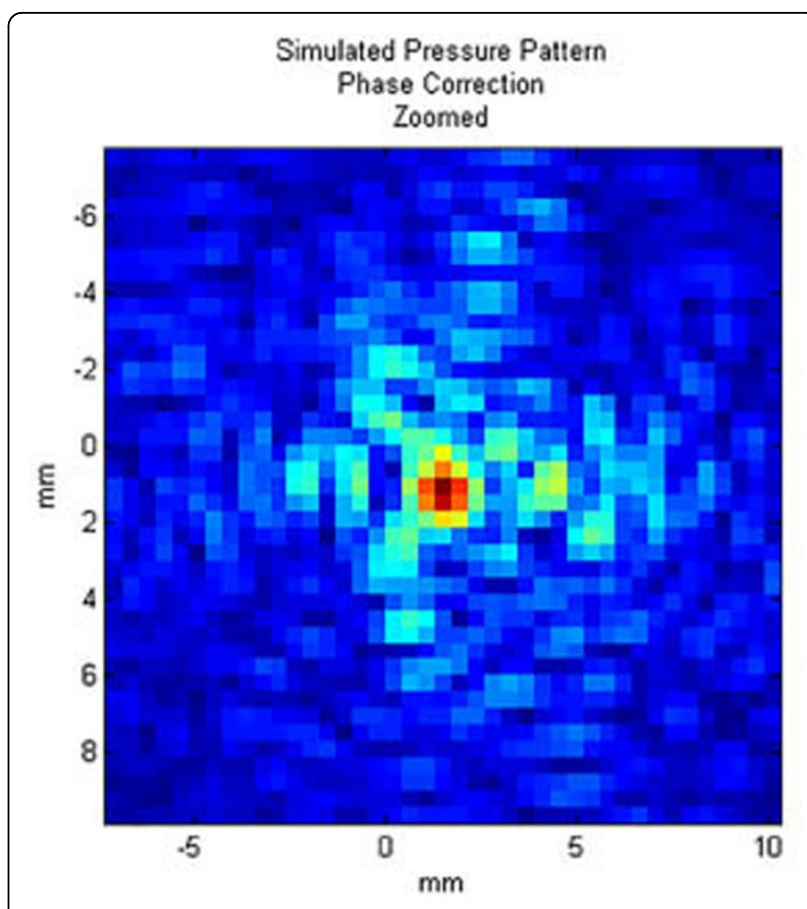

Figure 4 Zoomed-in view of focal spot for pressure pattern shown in 2 a.

There is an increase of approximately $25 \%$ in peak intensity when phase correction is employed.

\section{Acknowledgements (Funding)}

The authors thank Eyal Zadicario and Gilat Schiff of InSightec for providing data used in these simulations. This work was funded by NIH grant 5R01EB013433-03 and by the Focused Ultrasound Foundation.

Published: 30 June 2015

\section{References}

1. Vyas U, Christensen D: Ultrasound beam simulations in inhomogeneous tissue geometries using the hybrid angular spectrum method. Trans. UFFC 2012, 59(6):1093-1100.

2. Pichardo S, Sin V, Hynynen K: Multi-frequency characterization of the speed of sound and attenuation coefficient for longitudinal transmission of freshly excised human skulls. Phys. Med. Biol 2011, 56(1):219.

doi:10.1186/2050-5736-3-S1-P9

Cite this article as: Almquist et al: Simulation of hemispherical transducers for transcranial HIFU treatments using the hybrid angular spectrum approach. Journal of Therapeutic Ultrasound 2015 3(Suppl 1):P9. 